\title{
The Nearest Neighbor Nuclei Method to Objectify Analysis of Pertussis Toxin-Induced Clustering
}

\author{
Marieke E. Hoonakker ${ }^{1}$, Ed Remarque ${ }^{2}$, Jennifer Veth ${ }^{3}$, Arjen Sloots ${ }^{1}$ and Jeffrey J. Bajramovic ${ }^{3}$ \\ ${ }^{1}$ Institute for Translational Vaccinology (Intravacc), Bilthoven, The Netherlands; ${ }^{2}$ Department of Virology, Biomedical Primate Research Centre, Rijswijk, \\ The Netherlands; ${ }^{3}$ Alternatives Unit, Biomedical Primate Research Centre, Rijswijk, The Netherlands
}

\begin{abstract}
The in vivo histamine sensitization test (HIST) has historically been performed to guarantee the safety of acellular pertussis vaccine batches. Non-compliance of batches is primarily associated with the presence of low levels of pertussis toxin (PTx). Because of ethical, standardization and scientific reasons, a variety of alternative in vitro approaches have been studied to replace the lethal HIST. A broadly applied and partially accepted method is the CHO cell clustering test, which is based on the clustered growth pattern of $\mathrm{CHO}$ cells when exposed to minute amounts of PTx. One of the major hurdles for global application of the $\mathrm{CHO}$ clustering test is the manual assessment of the clusters, which is associated with suboptimal reproducibility of test outcomes and is time-consuming. Here, various parameters of $\mathrm{CHO}$ cell nuclei were evaluated in search for a reliable, objective read-out parameter. We demonstrate that the distance between each nucleus and its nearest neighbor ( $3 \mathrm{~N}$ method) is the most suitable parameter to assess clustered cell growth. This method detects $2.8 \mathrm{mIU} \mathrm{PTx} / \mathrm{mL}$ and thereby complies with the requirement set for the sensitivity of the $\mathrm{CHO}$ clustering test based on visual reading. In commercial acellular pertussis vaccines spiked with PTx, the method detects $45 \mathrm{mlU} / \mathrm{mL} P T x$, which is substantially lower than the $181-725 \mathrm{mlU} / \mathrm{mL}$ PTx detected by visual interpretation. The $3 \mathrm{~N}$ method thus allows objective and sensitive assessment of $\mathrm{CHO}$ clustering and thereby encourages broad and global implementation of the in vitro test as an alternative to the HIST.
\end{abstract}

\section{Introduction}

Pertussis toxoid (PTd) is the primary component of acellular pertussis $(\mathrm{aP})$ vaccines and is considered of high importance for vaccine-induced protective immunity (Sato et al., 1984; Black et al., 1988). PTd is generated by inactivation of pertussis toxin (PTx), a toxin that, even at low concentrations, is responsible for the occasional adverse effects of aP vaccines (Pittman, 1984; Tamura et al., 1982). PTx is an $\mathrm{AB}_{5}$ toxin, of which the B-oligomer binds to membrane-bound glycoproteins, upon which the toxin enters the cell and is transported to the Golgi and the endoplasmic reticulum. After dissociation from the B-oligomer, the A-subunit is released into the cytosol, where it ADP-ribosylates the $\alpha$-subunit of inhibitory $\mathrm{G}\left(\mathrm{G}_{\mathrm{i}}\right)$-coupled receptors (Plaut and Carbonetti, 2008; Katada et al., 1983). As a result, the $\alpha$-subunit can no longer inhibit its target enzyme adenylate cyclase (AC), which converts ATP into cAMP.

Monitoring reversion of the toxoid to its native form as well as assessment of residual PTx has long relied on the lethal murine histamine sensitization test (HIST), in which exposure to PTx in- creases the sensitivity to histamine by 30-300 times (Kind, 1958; Bergman and Munoz, 1965). This in vivo model, however, causes severe animal suffering, lacks mechanistic understanding, suffers from standardization problems, and accounts for the use of approximately 65,000 mice on a global level each year (Hoonakker et al., 2017).

The $\mathrm{CHO}$ cell clustering test is one of several alternatives to the HIST, in which assessment of PTx is based on the PTx-induced clustered growth of $\mathrm{CHO}$ cells (Xing et al., 2002; Hewlett et al., 1983). The CHO clustering test is widely applied as a routine test for aP bulk products. Utilization of this test for final aP products has long been hampered by the cytotoxic effect of aluminum salts in the product, an effect that can be circumvented by using semipermeable membranes (Isbrucker et al., 2016). In addition, the requirement to perform the test on the formulated final product has recently been waived by the European Pharmacopoeia (Ph. Eur.) (2020a), though it is still required in other regions of the world for these vaccines. Thus, broad and global implementation of the $\mathrm{CHO}$ cell clustering test still has to be achieved. One of the major hurdles is the manual reading of the
Received December 17, 2020; Accepted October 7, 2021;

Epub October 15, 2021; @ The Authors, 2022.

ALTEX 39(1), 140-148. doi:10.14573/altex.2012171

Correspondence: Marieke E. Hoonakker, PhD

Institute for Translational Vaccinology (Intravacc)

Bilthoven, The Netherlands

(Marieke_Esther@hotmail.com)
This is an Open Access article distributed under the terms of the Creative Commons Attribution 4.0 International license (http://creativecommons.org/licenses/by/4.0/) which permits unrestricted use, distribution and reproduction in any medium, provided the original work is appropriately cited. 
clustering, which is time-consuming and associated with variable test outcomes (Markey et al., 2019).

Clustered CHO cells form tightly packed groups resembling a rosette and are typically characterized by a more round morphology compared to unclustered cells (Hewlett et al., 1983). We hypothesized that these changes may be reflected in the characteristics of the nuclei, which are relatively easy to visualize and measure. We evaluated whether nuclear characteristics were altered as a result of exposure to PTx by analyzing DAPI-stained nuclei. The distance between neighboring nuclei was the only parameter that consistently corresponded with clustering and was therefore selected for further analysis. Using this parameter, we optimized the analysis method and determined the sensitivity to detect PTx alone as well as in the context of two commercial aP containing multivalent vaccines. Our findings provide proof-of-principle that measuring the distance between each nucleus and its single most adjacent nucleus is a sensitive and objective parameter. By objectifying the conventional subjective reading, the $3 \mathrm{~N}$ method might complement the current strategy to waive the HIST and encourage broad application of the $\mathrm{CHO}$ cell clustering test as an in vitro alternative.

\section{Materials and methods}

\section{Cell line and culture conditions}

Chinese hamster ovary (CHO-K1) cells purchased from ECACC (catalogues number 85051005 , lot $12 \mathrm{G} 006$, passage number $\mathrm{P}+7)$ were cultured $\left(37^{\circ} \mathrm{C}, 5 \% \mathrm{CO}_{2}\right)$ in F-12 Ham's (Sigma Aldrich) supplemented with $10 \%$ fetal calf serum (v/v), $100 \mathrm{U} / \mathrm{mL}$ penicillin, $100 \mu \mathrm{g} / \mathrm{mL}$ streptomycin, $0.3 \mathrm{mg} / \mathrm{mL}$ L-glutamine (Gibco). Fetal calf serum was used to adhere to the original clustering protocol (Isbrucker et al., 2016), though replacement of this medium component is envisaged in the future. Cells were passaged every 2-4 days using trypsin-EDTA, and cells of passage numbers 12-20 were used for the experiments.

\section{CHO cell clustering test}

Clustering was studied by plating $0.5 \mathrm{~mL} \mathrm{CHO}$ cells $\left(3 \times 10^{4}\right.$ cells $/ \mathrm{mL}$ or as indicated) in 24-well plates for $1 \mathrm{~h}$ at the bench at room temperature and subsequently for $2 \mathrm{~h}$ in an incubator at $37^{\circ} \mathrm{C}, 5 \% \mathrm{CO}_{2}$. Next, $250 \mu \mathrm{L}$ medium was removed and replaced by $250 \mu \mathrm{L}$ medium or aP vaccine containing the indicated concentrations of PTx (BRP1 cat\#Y0000021; the reference preparation of the European Directorate for the Quality of Medicines $\&$ Health Care (EDQM)) for $48 \mathrm{~h}$ at $37^{\circ} \mathrm{C}, 5 \% \mathrm{CO}_{2}$. One dilution of each concentration was prepared and added to three wells. Cells were fixed with methanol, and the nuclei were stained with DAPI (Sigma). CHO cell clustering was visualized using a Leica DMi6000B microscope at a magnification of 200x. 10x10 images of each well were obtained and stitched using LasX software. 24-well inserts $(0.4 \mu \mathrm{m}$, Pore Polycarbonate Membrane, Corning) were used when cells were exposed to vaccine. The PTx concentrations in the samples (and not the final concentration) are expressed in units as assigned for the $\mathrm{CHO}$ clustering test, one vial containing $50 \mu \mathrm{g}$ of PTx corresponding to $1360 \mathrm{IU}$ of CHO units (or $7500 \mathrm{IU}$ of HIST units). Two DTaP-IP (diphtheria, tetanus, acellular pertussis and inactivated polio) vaccines were purchased from Orly Pharma, i.e., Adacel from Sanofi containing $\mathrm{AlPO}_{4}$ and Pediarix from GSK containing $\mathrm{Al}(\mathrm{OH})$ and $\mathrm{AlPO}_{4}$. The vaccines were spiked with the indicated concentrations of PTx (BRP1) for $1 \mathrm{~h}$ at $4^{\circ} \mathrm{C}$ on an orbital shaker and centrifuged at $2000 \mathrm{rpm}$ for $10 \mathrm{~min}$ at $4^{\circ} \mathrm{C}$. The supernatant was discarded to clear its cytotoxic components, and the pellet fraction was resuspended in an equal amount of culture medium and stored over night at $4^{\circ} \mathrm{C}$ until use.

\section{Analysis of images}

After DAPI staining, the following protocol was used to analyze the images: 10x10 images were obtained and stitched. Using FIJI software, the Gaussian blur filter (tab ANALYZE) was applied with a value of 2.0 (to fill up large gaps in DAPI-stained nuclei). Background noise (see tab ANALYZE) was subtracted using a value of 50 (Untick light background). Measurement parameters were set (tab ANALYZE tick Area, Centroid, Perimeter, Area fraction). Type 8-bit (tab IMAGE) was chosen. Subsequently, the threshold was checked and adapted if necessary (tab IMAGE > Adjust > Threshold). It was confirmed that "Dark Background" was ticked. The used threshold was documented (normally between 10 and 20). Obtained images were adjusted to binary images (tab PROCESS $>$ Binary $>$ Make Binary), remaining holes (tab PROCESS $>$ Binary $>$ Fill holes) were filled, and overlapping nuclei were separated (tab PROCESS $>$ Binary $>$ Watershed). Characteristics and positions of the nuclei were analyzed (tab ANALYZE > Analyze particles and use the following settings: choose Size 250-10,000, Circularity 0.3-1, Outlines, and tick Pixels, and Clear results). Distances between nuclei were analyzed using either of the following plugins: $\mathrm{Nnd}^{1}$ and Simple $\mathrm{KNN}^{2}$ (see Text $\mathrm{S1}^{3}$ ). For simple KNN analysis, the distance between one or more neighboring nuclei based on their $\mathrm{X}$ and $\mathrm{Y}$ coordinates is determined. The results of this analysis were used for the figures shown here. The $\mathrm{NnD}$ is a simpler script that can only measure the distance between one nucleus and its most adjacent nucleus. $\mathrm{NnD}$ analysis and simple $\mathrm{KNN}$ analysis were saved as Excel files.

\section{Statistical analysis}

Statistical analysis of nuclear distances was performed with Microsoft R-Open version 3.5.0 (script is provided in Text $\mathrm{S}^{3}$ ). Nuclear distances and the other nuclear parameters (Tab. 1) were $\log 2$-transformed to obtain normality. Results are presented as the

\footnotetext{
1 https://icme.hpc.msstate.edu/mediawiki/index.php/Nearest_Neighbor_Distances_Calculation_with_ImageJ.html (accessed in 2019)

2 https://gist.github.com/lacan/2643f2ce7e33d1bb07adafde9ff94101 (accessed in 2017)

3 doi:10.14573/altex.2012171s
} 
Tab. 1: CHO cell clustering and the associated changes in DAPI stained nuclei Number: the number of nuclei detected in the analyzed field. Roundness (Round): $4 \times\left((\right.$ area $\left.) /\left(\pi \times(\text { major axis })^{2}\right)\right)$. Aspect ratio $(A R)$ : The aspect ratio of the particle's fitted ellipse, here (major axis)/(minor axis). Circularity (Circ.): $4 \pi \times\left((\right.$ area $\left.) /(\text { perimeter })^{2}\right)$ with a value of 1 indicating a perfect circle. As the value approaches 0 , this indicates an increasingly elongated shape. Area: area of selection (= nucleus) in square pixels. Distance: distance between two closest adjacent selections (= nucleus). The geometric mean of the nuclei in three wells, the confidence interval, and the corresponding percentage change compared to $0 \mathrm{mlU} / \mathrm{mL}$ PTx are shown. The table is based on one out of two representative experiments. *, significantly different from $0 \mathrm{IU} / \mathrm{ml} \mathrm{PTx;} \mathrm{vs,} \mathrm{versus;} \mathrm{AU,} \mathrm{arbitrary} \mathrm{units;} \mathrm{IU,} \mathrm{international} \mathrm{units}$

\begin{tabular}{|c|c|c|c|c|c|c|c|c|c|c|c|c|c|}
\hline \multirow{2}{*}{$\begin{array}{l}\begin{array}{l}\text { PTx } \\
(\mathrm{mlU} / \mathrm{mL})\end{array} \\
181\end{array}$} & \multirow{2}{*}{$\begin{array}{l}\begin{array}{l}\text { Growth } \\
\text { pattern }\end{array} \\
\text { Clustered }\end{array}$} & \multicolumn{2}{|l|}{ Number } & \multicolumn{2}{|c|}{ Round (AU) } & \multicolumn{2}{|c|}{ AR (AU) } & \multicolumn{2}{|c|}{ Circularity (AU) } & \multicolumn{2}{|c|}{ Area $(A U)$} & \multicolumn{2}{|c|}{ Distance (AU) } \\
\hline & & $\begin{array}{l}5884 \\
(4790- \\
7228)\end{array}$ & $3 \%$ & $\begin{array}{l}0.662 \\
(0.660- \\
0.664)\end{array}$ & $-2 \%$ & $\begin{array}{l}1.51 \\
(1.51- \\
1.52)\end{array}$ & $2 \%$ & $\begin{array}{l}0.812 \\
(0.811- \\
0.813)\end{array}$ & $-4 \%$ & $\begin{array}{l}0.208 \\
(0.207- \\
0.209)\end{array}$ & $1 \%$ & $\begin{array}{l}8.17 \\
(8.13- \\
8.20)\end{array}$ & $-19 \%$ \\
\hline 45 & Clustered & $\begin{array}{l}5227 \\
(3919- \\
6971)\end{array}$ & $-8 \%$ & $\begin{array}{l}0.679 \\
(0.677- \\
0.681)\end{array}$ & $1 \%$ & $\begin{array}{l}1.47 \\
(1.47- \\
1.48)\end{array}$ & $-1 \%$ & $\begin{array}{l}0.822 \\
(0.821- \\
0.823)\end{array}$ & $-3 \%$ & $\begin{array}{l}0.219 \\
(0.217- \\
0.220)\end{array}$ & $6 \%$ & $\begin{array}{l}8.30 \\
(8.26- \\
8.34)\end{array}$ & $-18 \%$ \\
\hline 11 & Clustered & $\begin{array}{l}6563 \\
(5940- \\
7251)\end{array}$ & $15 \%$ & $\begin{array}{l}0.669 \\
(0.667- \\
0.671)\end{array}$ & $-1 \%$ & $\begin{array}{l}1.50 \\
(1.49- \\
1.50)\end{array}$ & $1 \%$ & $\begin{array}{l}0.832 \\
(0.831- \\
0.832)\end{array}$ & $-1 \%$ & $\begin{array}{l}0.217 \\
(0.216- \\
0.218)\end{array}$ & $5 \%$ & $\begin{array}{l}8.88 \\
(8.83- \\
8.92)\end{array}$ & $-12 \%$ \\
\hline 3 & Clustered & $\begin{array}{l}6548 \\
(5186- \\
8269)\end{array}$ & $15 \%$ & $\begin{array}{l}0.672 \\
(0670- \\
0.674)\end{array}$ & $0 \%$ & $\begin{array}{l}1.49 \\
(1.49- \\
1.49)\end{array}$ & $1 \%$ & $\begin{array}{l}0.841 \\
(0.840- \\
0.842)\end{array}$ & $0 \%$ & $\begin{array}{l}0.225 \\
(0.224- \\
226)\end{array}$ & $9 \%$ & $\begin{array}{l}9.75 \\
(9.70- \\
9.79)\end{array}$ & $-4 \%$ \\
\hline 0 & Normal & $\begin{array}{l}5686 \\
(4539- \\
7122)\end{array}$ & & $\begin{array}{l}0.675 \\
(0.673- \\
0.677)\end{array}$ & & $\begin{array}{l}1.48 \\
(1.48- \\
1.49)\end{array}$ & & $\begin{array}{l}0.844 \\
(0.843- \\
0.844)\end{array}$ & & $\begin{array}{l}0.206 \\
(0.205- \\
0.207)\end{array}$ & & $\begin{array}{l}10.11 \\
(10.06- \\
10.17)\end{array}$ & \\
\hline
\end{tabular}

geometric mean of nuclear distance with corresponding $95 \%$ confidence intervals. The log2-transformed nuclear distance for each PTx concentration was compared to medium using a two-sample two-sided t-test, and differences were considered significant when $\mathrm{p}<0.05$. The Bonferroni method was used for correction of multiple comparisons. Unless mentioned otherwise, the results are expressed as the ratio of the nuclear distances (PTx/medium) with corresponding $95 \%$ confidence intervals (where 1 indicates no difference in nucleus distance and values $<1$ indicate clustering). Because a log transformation was applied, the estimated difference in nuclear distance is $\log 2(\mathrm{PTx})-\log 2$ (medium); the anti$\log$ of this estimate is a ratio as $\log a-\log b=\log a / b$.

The number of nuclei required to detect a specified geometric ratio of PTx/medium was calculated using the following formula:

$$
N=2 \times\left(\mathrm{Z}_{\alpha / 2}+\mathrm{Z}_{\beta}\right)^{2} \times\left(\frac{\mathrm{SD}}{\mathrm{ES}}\right)^{2}
$$

where $\mathrm{N}=$ required number of nuclei; $\mathrm{Z}_{\alpha / 2}=$ critical value for type $I$ error $=1.96(\mathrm{a}=0.05) ; Z_{\beta}=$ critical value for type II error $=2.33(1-\mathrm{b}=0.99)$; ES = effect size, expressed as log2(ratio); $\mathrm{SD}=$ standard deviation estimate based on three experiments.

\section{Results}

\subsection{Selection of parameters to assess clustering}

$\mathrm{CHO}$ cell nuclei were stained with DAPI (Fig. 1), and acquired images were converted to binary files. These files were used to evaluate nuclear size, morphology, and the distance between ad- jacent nuclei. While non-exposed cells and nuclei were randomly distributed (Fig. 1A), the distribution of both PTx-exposed cells as well as their DAPI-stained nuclei was characterized by a clustered pattern (Fig. 1B). Exposure to increasing PTx concentrations was associated with a decrease in overall cell numbers, but the high variability impeded the detection of significant effects (Tab. 1). The morphology of the nuclei (roundness and aspect ratio) was slightly but significantly altered upon exposure to 2.8 , 11 and $181 \mathrm{mIU} / \mathrm{mL}$ PTx (Tab. 1), while $45 \mathrm{mIU} / \mathrm{mL}$ PTx had no effect. In addition, nuclear circularity was slightly reduced when cells were exposed to PTx down to a concentration of $11 \mathrm{mIU} /$ $\mathrm{mL}$ (Tab. 1). Assessment of nuclear sizes (area) demonstrates that these were significantly increased when cells had been exposed to PTx with a detection level of $2.8 \mathrm{mIU} / \mathrm{mL} \mathrm{PTx}$, but there was no clear dose-response effect (Tab. 1), and the distribution was not normal (Fig. 1C). Most importantly, the distance between one nucleus and its nearest neighboring nucleus was significantly reduced when cells were exposed to PTx down to a concentration of $2.8 \mathrm{mIU} / \mathrm{mL}$ (Tab. 1). The percentage change in this distance increased with increasing PTx concentrations (Tab. 1) and was normally distributed, though slightly skewed towards the right (Fig. 1D). Consequently, the distance between nearest neighboring nuclei was selected as the most promising parameter.

\subsection{The effect of combining triplicate measures versus individual measures}

The analysis of the PTx reference and vaccine bulk samples in monograph 2.6.33 of the Ph. Eur. on "residual pertussis toxin" is based on at least dual measurements. In Figure 1 and Table 1, 

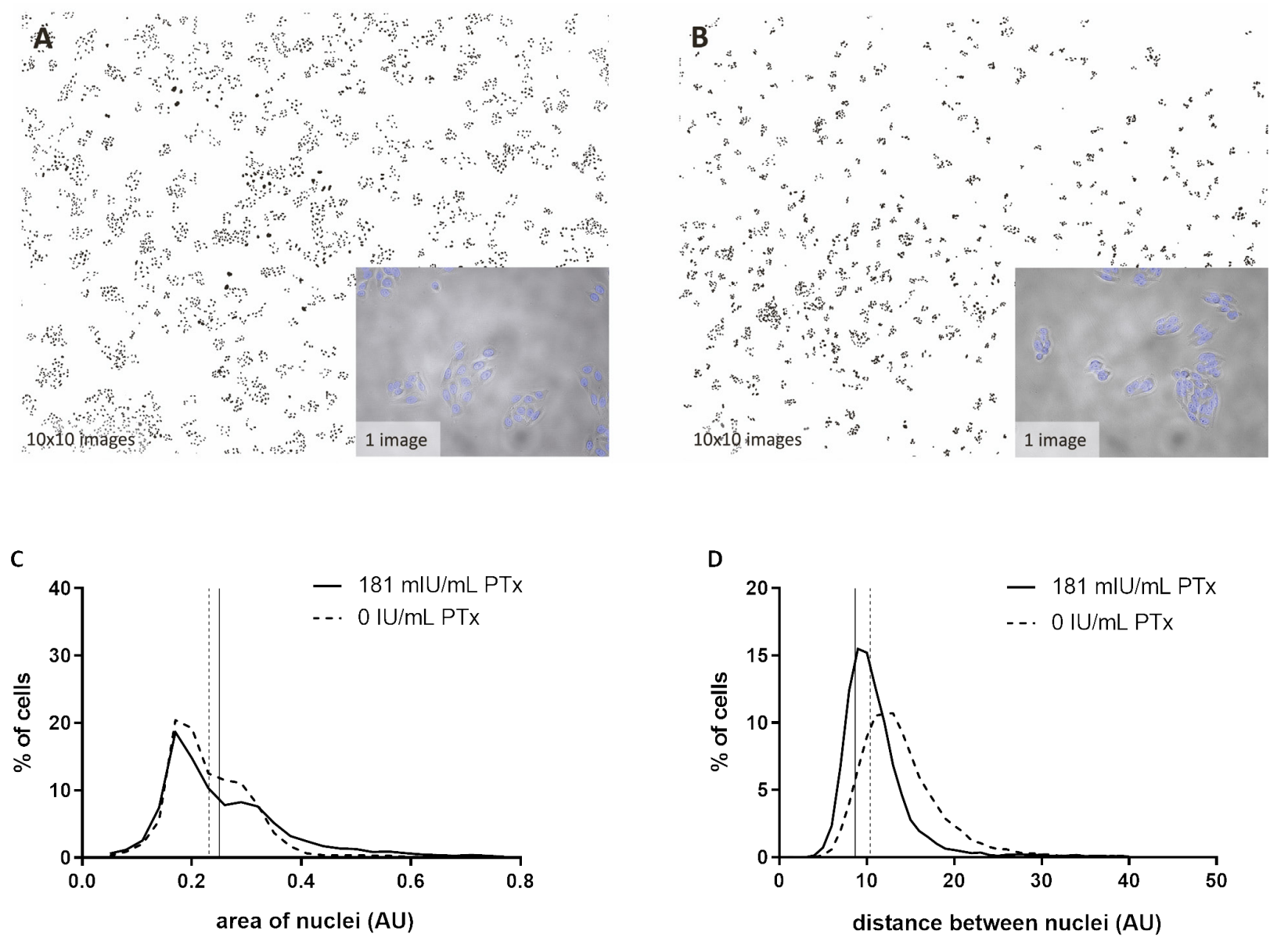

Fig. 1: The effect of PTx on the nuclear distribution of $\mathrm{CHO}$ cells

$\mathrm{CHO}$ cells were exposed to medium (A) or $181 \mathrm{mIU} / \mathrm{mL} \mathrm{PTx} \mathrm{(B)} \mathrm{for} 48 \mathrm{~h}$, and nuclei were visualized with DAPI (shown in blue/purple). Fluorescence images were converted to binary images. Examples of one overlay (fluorescence and light microscopical, 1x image) of each exposure condition and corresponding stitched binary images (composed of $10 \times 10$ images) are shown. Based on the analysis of all nuclei in three wells, the area of the nuclei $(C)$ and the distance between the nearest neighboring nuclei (D) were determined. One out of two representative experiments is shown. The lines indicate the geometric mean of three wells for each condition. AU, arbitrary units

statistical analysis was performed by combining all nuclei in three wells (i.e., triplicate measures) to ensure optimal statistical resolution. Since analysis of the nuclei in one well (i.e., single measures) might be more practical, statistical analyses were performed either by combined analysis of the triplicates or by performing the analysis on single measures. In both the triplicate (Fig. 2A; Fig. S1A, ${ }^{3}$ ) as well as individual analysis (Fig. 2B; Fig. S1C, $\mathrm{D}^{3}$ ), the effect of PTx was either pronounced (11$725 \mathrm{mIU} / \mathrm{mL})$ or slight $(2.8 \mathrm{mIU} / \mathrm{mL})$. The individual analysis is associated with substantial variation between triplicates, which is compensated by combining the statistical analyses of triplicates. We therefore consider combined analysis as optimal for PTX-induced clustering analysis.

\subsection{Nuclear numbers required for reliable analyses}

The minimal number of cells required for reliable analysis was evaluated statistically (Fig. 2C) and experimentally (Fig. S2 ${ }^{3}$ ). Figure $2 \mathrm{C}$ shows that the total number of nuclei required for reliable analysis is 302 nuclei for PTx in the concentration range of $11-725 \mathrm{IU} / \mathrm{mL}$ and 6294 nuclei for a concentration of $2.8 \mathrm{mIU} /$ $\mathrm{mL}$. The average number of nuclei in each binary image is approximately 3500 , explaining the necessity to combine the analysis of triplicates for detection of $2.8 \mathrm{mIU} / \mathrm{ml}$ PTx. When we experimentally halved cell numbers (Fig. S2 $\mathrm{A}^{3}$ ), the ratios PTx/medium were slightly enhanced (average ratio PTx/medium was 0.90 instead of 0.86 ), but the lowest detectable PTx concentration remained $2.8 \mathrm{mIU} / \mathrm{mL}$. In contrast, experimentally increasing cell 


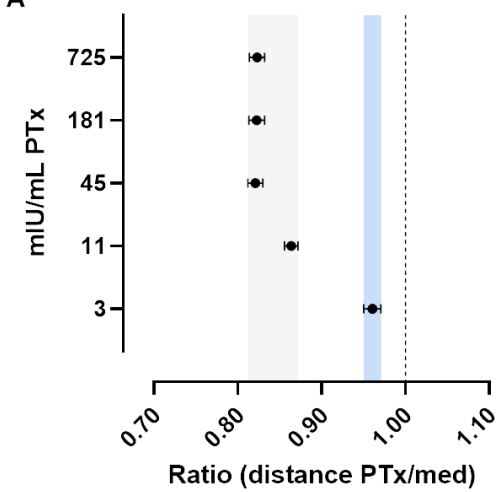

B

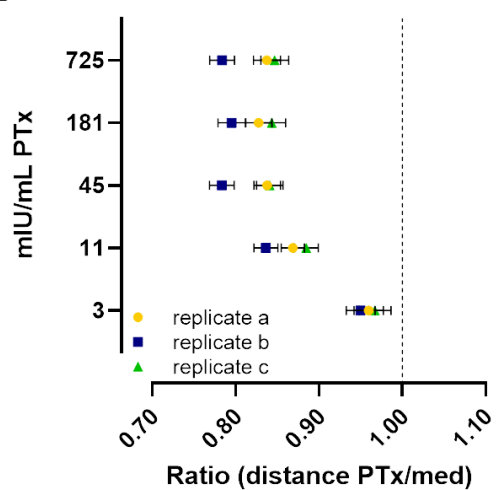

C

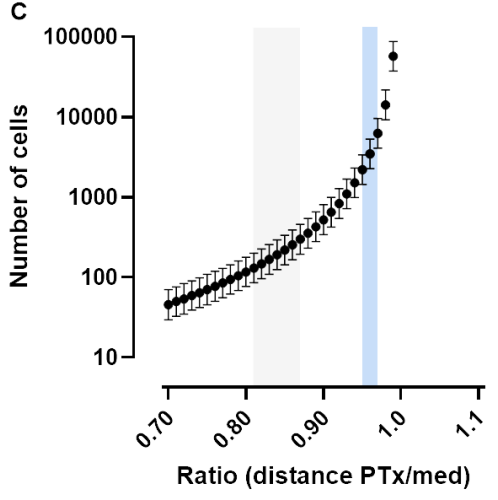

Fig. 2: The distance between nearest neighboring nuclei using triplicates or single measurements

$\mathrm{CHO}$ cells were exposed to $0,2.8,11,45,181$ and $725 \mathrm{mlU} / \mathrm{mL}$ PTx for $48 \mathrm{~h}$, and nuclei were visualized with DAPI. Fluorescence images were acquired, stitched ( $10 \times 10$ images), and converted to binary images. The ratio of the geometric mean distance between nuclei for the indicated PTx concentration divided by the geometric mean distance between nuclei for medium and the confidence interval for each concentration of PTx are shown. Analysis was based on all nuclei in three wells (A) or on the nuclei in the individual wells (B). One out of three representative experiments is shown; both other experiments are shown in Figure $S 1^{3}$. (C) The number of nuclei required for reliable analysis for each ratio of $\mathrm{PTx} /$ medium, based on three experiments. Highlighted in grey $(\mathrm{A}, \mathrm{C})$ is the range of ratios for $11-725 \mathrm{IU} / \mathrm{mL} \mathrm{PTx}$ and in blue the ratio for $2.8 \mathrm{IU} / \mathrm{mL} \mathrm{PTx}$.

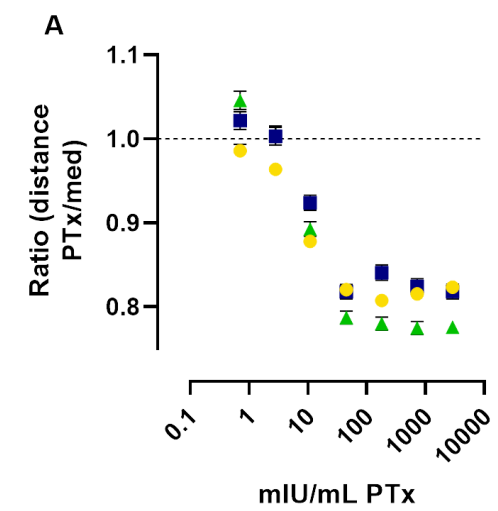

C

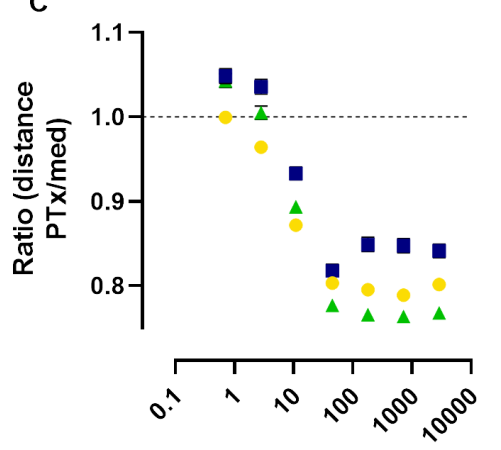

$\mathrm{mIU} / \mathrm{mL} \mathrm{PTx}$

\section{B}

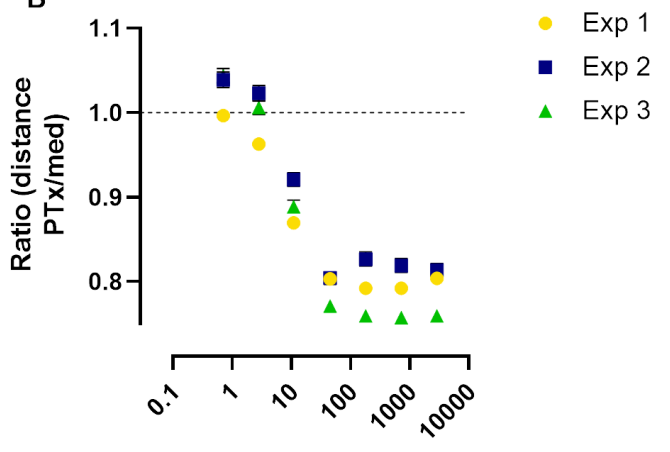

$\mathrm{mIU} / \mathrm{mL}$ PTx

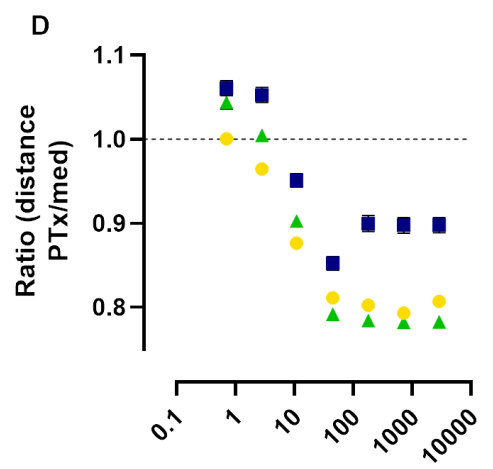

$\mathrm{mIU} / \mathrm{mL}$ PTx
Fig. 3: Statistical analysis of the distance to one, two, three or four nearest neighboring nuclei

$\mathrm{CHO}$ cells were exposed to $0,0.7$, 2.8, 11, 45, 181, 725 and 2901mIU/ $\mathrm{mL}$ PTx for $48 \mathrm{~h}$, and nuclei were visualized with DAPI. Fluorescent images were acquired, stitched (10 $\mathrm{x}$ 10 images) and converted to binary images. The ratio of the geometric mean distance between nuclei for the indicated PTx concentration divided by the geometric mean distance between nuclei for medium for one $(A)$, two $(B)$, three $(C)$ or four (D) nearest neighboring nuclei is shown. Results of three independent experiments (Exp 1, 2 and 3 ) and the confidence interval for each concentration of PTx are shown. The black lines indicate a ratio of 1 . 
Tab. 2: Reproducibility using selected conditions

IU, international units; AU, arbitrary units; low, lower; upp, upper; ratio, geometric mean distance between one nucleus and its most adjacent nucleus for the indicated PTx concentration divided by the geometric mean distance between nuclei for medium.

\begin{tabular}{|c|c|c|c|c|c|c|c|c|c|}
\hline PTx (mlU/mL) & & 0 & 2.8 & 3.8 & 5.7 & 11 & 45 & 181 & 725 \\
\hline \multirow[t]{2}{*}{ Experiment 1} & $\begin{array}{l}\text { AU } \\
\text { (low - upp) }\end{array}$ & $\begin{array}{l}9.41 \\
(9.31- \\
9.51)\end{array}$ & $\begin{array}{l}9.14 \\
(9.02- \\
9.26)\end{array}$ & $\begin{array}{l}8.95 \\
(8.83- \\
9.07)\end{array}$ & $\begin{array}{l}8.65 \\
(8.56- \\
8.75)\end{array}$ & $\begin{array}{l}8.69 \\
(8.56- \\
8.82)\end{array}$ & $\begin{array}{l}8.17 \\
(8.04- \\
8.31)\end{array}$ & $\begin{array}{l}8.28 \\
(8.14- \\
8.41)\end{array}$ & $\begin{array}{l}8.18 \\
(8.05- \\
8.31)\end{array}$ \\
\hline & $\begin{array}{l}\text { Ratio } \\
\text { (low - upp) }\end{array}$ & - & $\begin{array}{l}0.97 \\
(0.99- \\
0.95)\end{array}$ & $\begin{array}{l}0.95 \\
(0.97- \\
0.94)\end{array}$ & $\begin{array}{l}0.92 \\
(0.93- \\
0.91)\end{array}$ & $\begin{array}{l}0.92 \\
(0.94- \\
0.91)\end{array}$ & $\begin{array}{l}0.87 \\
(0.89- \\
0.85)\end{array}$ & $\begin{array}{l}0.88 \\
(0.90- \\
0.86)\end{array}$ & $\begin{array}{l}0.87 \\
(0.89- \\
0.85)\end{array}$ \\
\hline \multirow[t]{2}{*}{ Experiment 2} & $\begin{array}{l}\text { AU } \\
\text { (low - upp) }\end{array}$ & $\begin{array}{l}10.04 \\
(9.98- \\
10.11)\end{array}$ & $\begin{array}{l}9.65 \\
(9.57- \\
9.73)\end{array}$ & $\begin{array}{l}9.69 \\
(9.61- \\
9.76)\end{array}$ & $\begin{array}{l}9.70 \\
(9.62- \\
9.78)\end{array}$ & $\begin{array}{l}8.67 \\
(8.62- \\
8.73)\end{array}$ & \begin{tabular}{|l}
8.24 \\
$(8.17-$ \\
$8.31)$
\end{tabular} & $\begin{array}{l}8.26 \\
(8.18- \\
8.33)\end{array}$ & $\begin{array}{l}8.26 \\
(8.19- \\
8.34)\end{array}$ \\
\hline & $\begin{array}{l}\text { Ratio } \\
\text { (low - upp) }\end{array}$ & - & $\begin{array}{l}0.96 \\
(0.95- \\
0.97)\end{array}$ & $\begin{array}{l}0.96 \\
(0.95- \\
0.97)\end{array}$ & $\begin{array}{l}0.97 \\
(0.96- \\
0.98)\end{array}$ & $\begin{array}{l}0.86 \\
(0.86- \\
0.87)\end{array}$ & $\begin{array}{l}0.82 \\
(0.81- \\
0.83)\end{array}$ & $\begin{array}{l}0.82 \\
(0.81- \\
0.83)\end{array}$ & $\begin{array}{l}0.82 \\
(0.81- \\
0.83)\end{array}$ \\
\hline \multirow[t]{2}{*}{ Experiment 3} & $\begin{array}{l}\text { AU } \\
\text { (low - upp) }\end{array}$ & \begin{tabular}{|l|}
10.02 \\
$(9.97-$ \\
$10.07)$
\end{tabular} & $\begin{array}{l}9.55 \\
(9.50- \\
9.60)\end{array}$ & $\begin{array}{l}9.57 \\
(9.52- \\
9.63)\end{array}$ & $\begin{array}{l}9.26 \\
(9.21- \\
9.30)\end{array}$ & $\begin{array}{l}9.18 \\
(9.12- \\
9.23)\end{array}$ & $\begin{array}{l}8.30 \\
(8.26- \\
8.35)\end{array}$ & $\begin{array}{l}8.22 \\
(8.17- \\
8.27)\end{array}$ & $\begin{array}{l}8.14 \\
(8.09- \\
8.19)\end{array}$ \\
\hline & $\begin{array}{l}\text { Ratio } \\
\text { (low - upp) }\end{array}$ & - & $\begin{array}{l}0.95 \\
(0.95- \\
0.96)\end{array}$ & $\begin{array}{l}0.96 \\
(0.95- \\
0.96)\end{array}$ & $\begin{array}{l}0.92 \\
(0.92- \\
0.93)\end{array}$ & $\begin{array}{l}0.92 \\
(0.91- \\
0.92)\end{array}$ & $\begin{array}{l}0.83 \\
(0.82- \\
0.84)\end{array}$ & $\begin{array}{l}0.82 \\
(0.81- \\
0.83)\end{array}$ & $\begin{array}{l}0.81 \\
(0.81- \\
0.82)\end{array}$ \\
\hline
\end{tabular}

numbers (Fig. S2 $\mathrm{C}^{3}$ ) did not substantially influence the ratio PTx/ medium but slightly reduced the sensitivity to detect PTx $(11 \mathrm{mIU} /$ $\mathrm{mL}$ PTx instead of $2.8 \mathrm{mIU} / \mathrm{mL}$ PTx). These minimal effects of cell densities underline the robustness of the $3 \mathrm{~N}$ method..

\subsection{Analysis of one to four nearest neighboring nuclei}

Although the distance between one nucleus and its closest adjacent neighbor nucleus already enables detection of PTx levels as low as $2.8 \mathrm{mIU} / \mathrm{mL}$, analysis of the distance of each nucleus to two or more adjacent nuclei might improve the sensitivity of the test further (Fig. 3). By analyzing the distance of each nucleus to its single nearest neighbor, the lowest concentration detected was between 0.7 and $11 \mathrm{mIU} / \mathrm{mL}$ PTx, whereas the lowest concentration detected based on the analysis of the distance to its two, three or four nearest nuclei was between 2.8 and $11 \mathrm{mIU} / \mathrm{mL}$ PTx (Fig. $3)$. Repetitions of this experiment, which included several concentrations within the range of the lowest concentrations detected, revealed that analysis of an increasing number of nuclei led to an increase in variation towards the point that PTx effects were no longer significant for four nuclei in one out of three experiments (Fig. $\mathrm{S}^{3}$ ). In the other two experiments (Fig. S3 ${ }^{3}$ ), the ratio between cells treated with and without PTx was similar for one and for two nuclei but was reduced when higher numbers of neighboring nuclei were analyzed. Therefore, analysis of one or two nuclei is considered optimal. For practical reasons, one neighboring nucleus was analyzed in the subsequent experiments.

The three experiments provide an indication for the reproducibility depicted in more detail in Table 2 . There is some variability between the three experiments regarding the absolute distance and a slight variation for the ratios PTx/medium. However, in all

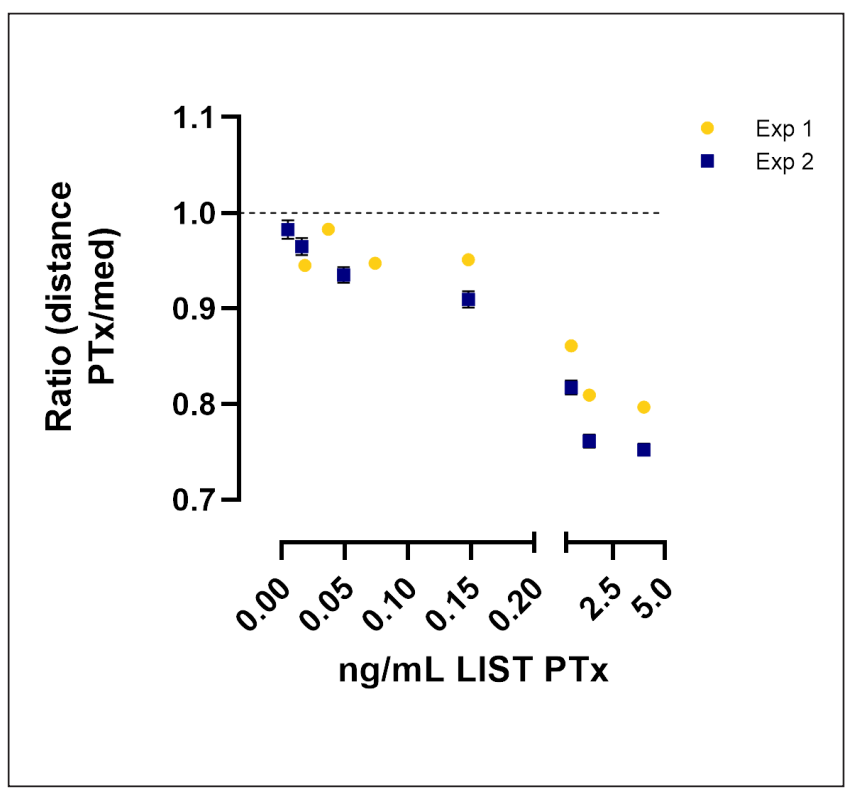

Fig. 4: CHO cell clustering in response to PTx of LIST Biological

CHO cells were exposed to 0 and $0.005-4.0 \mathrm{ng} / \mathrm{mL}$ PTx (LIST Biological) for $48 \mathrm{~h}$, and nuclei were visualized with DAPI. Fluorescent images were acquired, stitched $(10 \times 10)$ and converted to binary images. The ratio of the geometric mean distance between nuclei for the indicated PTx concentration divided by the geometric mean distance between nuclei for medium and the confidence interval for each concentration of PTx are shown. The black line indicates a ratio of 1 . 
A

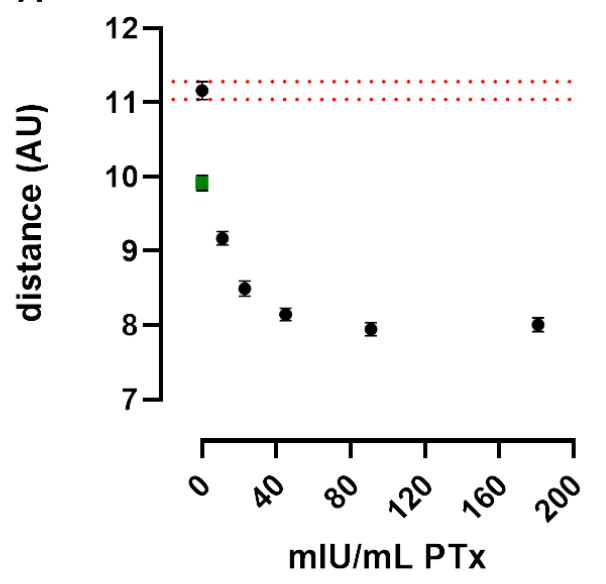

B

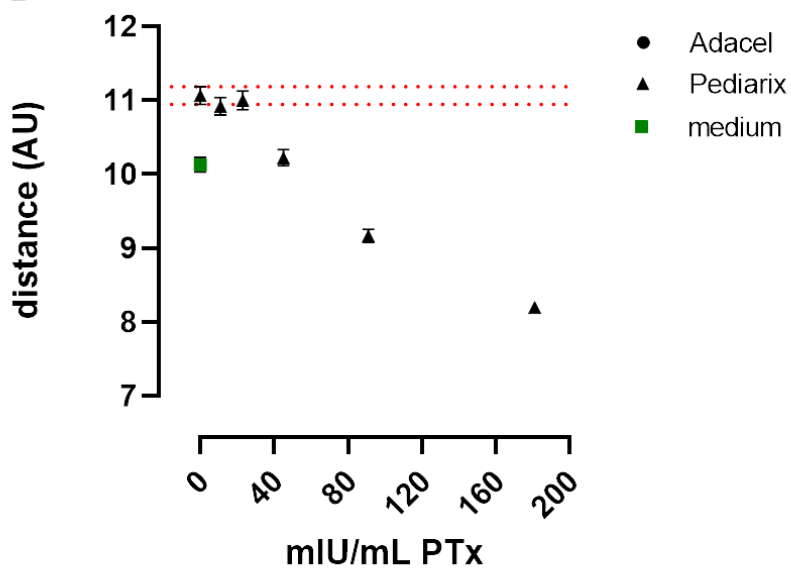

Fig. 5: The effect of PTx-spiked aP vaccines on the distance between each nucleus and its most adjacent nucleus Two DTaP-IP vaccines (Adacel from Sanofi containing $\mathrm{AIPO}_{4}(\mathrm{~A})$ and Pediarix from GSK containing $\mathrm{Al}(\mathrm{OH})$ and $\mathrm{AIPO}$ (B)) were spiked with $0,11,23,45,91$ and $181 \mathrm{mIU} / \mathrm{mL} \mathrm{PTx}$. CHO cells were exposed to these vaccine samples or medium for $48 \mathrm{~h}$, and nuclei were visualized with DAPI. Fluorescence microscopical images were acquired, stitched (10 x 10) and converted to binary images. The geometric mean distance between one nucleus and its nearest neighbor and the confidence interval for each concentration of PTx for one experiment are shown. The red lines indicate the confidence interval for $0 \mathrm{IU} / \mathrm{mL}$ PTx (in vaccine). AU, arbitrary units

three experiments, the $3 \mathrm{~N}$ method consistently detects a significant effect of 2.8-725 mIU/mL PTx. Nevertheless, formal assessment of the test's sensitivity and reproducibility need to be confirmed in a (pre)validation study.

\subsection{A second source of PTx}

Although the European PTx standard (BRP1) is commonly applied within and outside Europe, PTx is produced by many others, and the biological activities of these preparation are highly variable and do not necessarily correspond with their protein concentration. Therefore, compatibility of the $3 \mathrm{~N}$ method with other sources of PTx is of importance. One commonly used source is the PTx produced by LIST Biological, for which clustering based on visual reading has been detected at concentrations that were as low as $0.025-0.1 \mathrm{ng}$ PTx $/ \mathrm{mL}$ (Wagner et al., 2017). Using the $3 \mathrm{~N}$ method, clustering could be detected with concentrations as low as $0.005 \mathrm{ng}$ PTx/mL LIST PTx (Fig. 4).

\subsection{Analysis of PTx in aP vaccines}

Although the $\mathrm{Ph}$. Eur. waived the requirement to perform the in vivo HIST for testing of residual PTx and reversion to toxicity of all PTd and final products, the test is still stipulated for these vaccines in other regions of the world. Compliance of the method proposed in this study with formulated aP vaccines would therefore greatly facilitate broad and global application. Preliminary experiments showed that aP vaccine supernatant was cytotoxic to $\mathrm{CHO}$ cells and made a membrane necessary to prevent detrimental effects of the adjuvant (data not shown). Therefore, semipermeable membranes were applied and used to evaluate the pel- let fraction of two commercial aP vaccines, Adacel containing $\mathrm{AlPO}_{4}$ and Pediarix containing $\mathrm{Al}(\mathrm{OH})$ and $\mathrm{AlPO}_{4}$. The plain aP vaccines were not inert: They reduced cellular confluency and slightly increased the distance between the nuclei as compared to cells grown in medium alone (Fig. 5A,B), which might be the result of the adjuvant or adsorbed vaccine components. PTx in a concentration range of 11-181 and 45-181 mIU/mL spiked into Adacel and Pediarix, respectively, significantly reduced the distance between one nucleus and its most adjacent neighbor nucleus as compared to unspiked vaccine. The $3 \mathrm{~N}$ method was therefore capable of detecting lower concentrations of PTx than included in the collaborative study using visual inspection of clustering (Isbrucker et al., 2016). Depending on the laboratory and the vaccine, the lowest concentration detected in four aP vaccines was either 181,363 or $725 \mathrm{mIU}$ PTx $/ \mathrm{mL}$.

\section{Discussion}

The current $\mathrm{CHO}$ clustering test is based on visual assessment of the cellular growth pattern, which is labor-intensive and requires specific training. Due to its subjective nature, the method is inherently prone to intra- and interlaboratory variation (Xing et al., 2010), although recent studies showed significant improvement following application of an appropriate reference and a standardized protocol (Isbrucker et al., 2016; Markey et al., 2018). Despite attempts by different laboratories, a reliable and objective parameter for clustering has not yet been identified. When clustered, the $\mathrm{CHO}$ cell morphology changes, and the cells be- 
come more packed and form islets (Fig. 1). As nuclei are relatively easy to stain, we evaluated whether clustering was associated with changes in nuclear numbers, size, shape, and the distance between nuclei. Our analysis showed that the distance between nearest neighboring nuclei ( $3 \mathrm{~N}$ method) was the only parameter that consistently corresponded with clustering. Statistical evaluation of various factors demonstrates that analysis of the distance of each nucleus to its single most adjacent nucleus and combining the counts of cells in three wells is the best procedure to determine $\mathrm{CHO}$ clustering in an objective manner.

In principle, the $\mathrm{CHO}$ clustering test is a limit test for the detection of PTx in intermediate aP vaccine products, although evaluation of dilution ranges of products also allow for the approximative assignment of PTx concentrations (Ph. Eur., 2020b). Using our $3 \mathrm{~N}$ method, we show that the distance between nuclei of CHO cells is linear between 5.7 and $45 \mathrm{mIU} / \mathrm{mL}$ PTx (Fig. $\mathrm{S}^{3}$ ), although the range of this linear part varies somewhat between experiments (Tab. 2). We therefore recommend assessment of nuclear distances as a qualitative test. For such qualitative tests, the $\mathrm{ICH}$ requires the evaluation of the detection limit and the specificity (ICH, 2005). Using the European reference PTx standard (BRP1), the detection limit for the CHO clustering test based on visual examination is $5 \mathrm{mIU} / \mathrm{mL}$ (CHO units) (Ph. Eur., 2020b), while the lowest concentration detected with the $3 \mathrm{~N}$ method is $2.8 \mathrm{mIU} / \mathrm{mL}$ PTx. The source of PTx commonly used in the US is LIST Biological, for which the CHO clustering limit is $0.025-0.1 \mathrm{ng} / \mathrm{mL}$ PTx (Wagner et al., 2017), while it is $0.019 \mathrm{ng} / \mathrm{mL}$ PTx using the $3 \mathrm{~N}$ method. The European requirement for the sensitivity of the $\mathrm{CHO}$ clustering provides an important and central point of focus for the assay sensitivity using purified PTx. For final product testing, there is consensus that an in vitro alternative should have a sensitivity at least equivalent to the HIST (Arciniega et al., 2016; Wagner et al., 2016), which nevertheless poses a challenge due to the diverse sensitivities of regional variants of this in vivo test (Arciniega et al., 2016). Using the $\mathrm{Ph}$. Eur. method, the HIST has a detection limit of 1-2 IU (HIST units corresponding to 181-363 mIU CHO cell units) PTx in aP vaccines (Xing et al., 2010; Bache et al., 2012). Here, experiments with two commercial aP vaccines spiked with purified PTx demonstrate that the lowest detectable concentrations are as low as 11 and $45 \mathrm{IU} / \mathrm{mL}$ PTx for Adacel and Pediarix respectively when measuring the distance between nuclei. This is well below the sensitivity of the HIST and below the lowest PTx concentration included in a collaborative study based on visual reading of CHO clusters (Isbrucker et al., 2016). Some regulatory agencies require testing for residual PTx as well as reversion of PTd to its toxic forms (Hoonakker et al., 2017), and a follow-up validation study should therefore confirm the test's detection limit for both purposes using the $3 \mathrm{~N}$ method.

The other criterion of the $\mathrm{ICH}$, specificity, is described as "the ability to assess unequivocally the analyte in the presence of components which may be expected to be present" (ICH, 2005). The two vaccines used in this study were produced by Sanofi and GSK and differ in their matrix and adjuvant composition, factors known to affect CHO cell clustering (Isbrucker et al., 2016). Here, we show that the distance between nuclei increases as a re- sult of exposure to these commercial aP vaccines (Fig. 4). This is most likely the result of a decrease in cellular confluency caused by the adjuvant fraction or minute amounts of the diluent fraction that might be slightly toxic to the $\mathrm{CHO}$ cells, even when separated from the cells with a semipermeable membrane (Isbrucker et al., 2016). These slight but significant effects can be compensated by normalization using a safe batch of the same composition as the vaccine under study in each experiment, according to the principles of the consistency approach (De Mattia et al., 2011). The compliance of the $3 \mathrm{~N}$ method with both ICH criteria underscores the suitability of the method to assess clustered growth and thereby contributes to application of the $\mathrm{CHO}$ clustering test for final product assessment. Nevertheless, further research should include in-house validation studies, evaluating the method's reproducibility and sensitivity by using blinded, spiked samples. In that respect, the $3 \mathrm{~N}$ method could be applied side-byside with the conventional reading method.

We here show that the $3 \mathrm{~N}$ method is valid for assessing clustered growth of CHO cells. Scanning of the plates, preparation of the binary pictures, and the statistical analysis can and should be automated to reduce hands-on-time up to 2-4 fold. Our study provides proof-of-principle that measuring the distance between nearest neighboring nuclei is a sensitive and objective read-out for $\mathrm{CHO}$ cell clustering exposed to the different PTx preparations used in this study. This indicates that the method may be applied for bulk product testing within Europe, although it should be subjected to further in-house validation before application. Furthermore, we demonstrate the validity of this method for testing of final aP vaccine products, which could endorse implementation of the $\mathrm{CHO}$ clustering test as an alternative for the HIST on a global level.

\section{References}

Arciniega, J., Wagner, L., Prymula, R. et al. (2016). Alternatives to HIST for acellular pertussis vaccines: Progress and challenges in replacement. Pharmeur Bio Sci Notes 2016-04, 8296.

Bache, C., Hoonakker, M., Hendriksen, C. et al. (2012). Workshop on animal free detection of pertussis toxin in vaccines Alternatives to the histamine sensitisation test. Biologicals 40, 309-311. doi:10.1016/j.biologicals.2012.04.002

Bergman, R. K. and Munoz, J. (1965). Circulatory collapse in anaphylaxis and histamine toxicity in mice. J Immunol 95, 1-8.

Black, W. J., Munoz, J. J., Peacock, M. G. et al. (1988). ADPribosyltransferase activity of pertussis toxin and immunomodulation by Bordetella pertussis. Science 240, 656-659. doi:10.1126/science. 2896387

De Mattia, F., Chapsal, J. M., Descamps, J. et al. (2011). The consistency approach for quality control of vaccines - A strategy to improve quality control and implement 3Rs. Biologicals 39, 59-65. doi:10.1016/j.biologicals.2010.12.001

Hewlett, E. L., Sauer, K. T., Myers, G. A. et al. (1983). Induction of a novel morphological response in Chinese hamster ovary cells by pertussis toxin. Infect Immun 40, 1198-1203. doi:10.1128/iai.40.3.1198-1203.1983 
Hoonakker, M., Arciniega, J. and Hendriksen, C. (2017). Safety testing of acellular pertussis vaccines: Use of animals and 3Rs alternatives. Hum Vaccin Immunother 13, 2522-2530. doi:10. 1080/21645515.2017.1349585

ICH (2005). Q2(R1) Validation of Analytical Procedures: Text and Methodology. https://www.ema.europa.eu/en/ichq2-r1-validation-analytical-procedures-text-methodology

Isbrucker, R., Wagner, L., Daas, A. et al. (2016). Transferability study of CHO cell clustering assays for monitoring of pertussis toxin activity in acellular pertussis vaccines. Pharmeur Bio Sci Notes 2016-05, 97-114.

Katada, T., Tamura, M. and Ui, M. (1983). The A protomer of islet-activating protein, pertussis toxin, as an active peptide catalyzing ADP-ribosylation of a membrane protein. Arch Biochem Biophys 224, 290-298. doi:10.1016/0003-9861(83)90212-6

Kind, L. S. (1958). The altered reactivity of mice after inoculation with Bordetella pertussis vaccine. Bacteriol Rev 22, 173182. doi:10.1128/br.22.3.173-182.1958

Markey, K., Douglas-Bardsley, A., Hockley, J. et al. (2018). Calibration of pertussis toxin BRP batch 1 in a standardised $\mathrm{CHO}$ cell-based clustering assay. Pharmeur Bio Sci Notes 2018, 112-123.

Markey, K., Asokanathan, C. and Feavers, I. (2019). Assays for determining pertussis toxin activity in acellular pertussis vaccines. Toxins (Basel) 11, 417. doi:10.3390/toxins11070417

Ph. Eur. - European Pharmacopoeia (2020a). Pertussis vaccines (acellular, co-purified, adsorbed) 1595.

Ph. Eur. (2020b). Residual pertussis toxin 2.6.33.

Pittman, M. (1984). The concept of pertussis as a toxin-mediated disease. Pediatr Infect Dis 3, 467-486. doi:10.1097/00006454198409000-00019

Plaut, R. D. and Carbonetti, N. H. (2008). Retrograde transport of pertussis toxin in the mammalian cell. Cell Microbiol 10, 1130-1139. doi:10.1111/j.1462-5822.2007.01115.x

Sato, H., Ito, A., Chiba, J. et al. (1984). Monoclonal antibody against pertussis toxin: Effect on toxin activity and pertussis infections. Infect Immun 46, 422-428. doi:10.1128/ iai.46.2.422-428.1984
Tamura, M., Nogimori, K., Murai, S. et al. (1982). Subunit structure of islet-activating protein, pertussis toxin, in conformity with the A-B model. Biochemistry 21, 5516-5522. doi:10.1021/bi00265a021

Wagner, L., Isbrucker, R., Locht, C. et al. (2016). In search of acceptable alternatives to the murine histamine sensitisation test (HIST): What is possible and practical? Pharmeur Bio Sci Notes 2016, 151-170.

Wagner, L. D., Corvette, L. J., Ngundi, M. M. et al. (2017). Towards replacement of the acellular pertussis vaccine safety test: Comparison of in vitro cytotoxic activity and in vivo activity in mice. Vaccine 35, 7160-7165. doi:10.1016/j. vaccine.2017.10.082

Xing, D., Das, R. G., Newland, P. et al. (2002). Comparison of the bioactivity of reference preparations for assaying Bordetella pertussis toxin activity in vaccines by the histamine sensitisation and Chinese hamster ovary-cell tests: Assessment of validity of expression of activity in terms of protein concentration. Vaccine 20, 3535-3542. doi:10.1016/S0264410X(02)00338-9

Xing, D., Maes, A., Behr-Gross, M. E. et al. (2010). Collaborative study for the standardisation of the histamine sensitizing test in mice and the CHO cell-based assay for the residual toxicity testing of acellular pertussis vaccines. Pharmeur Bio Sci Notes 2010, 51-63.

\section{Conflict of interest}

The authors declare no financial or commercial conflicts of interest.

\section{Acknowledgements}

This study was part of a project funded by the Dutch Ministry of Health, Welfare and Sports and the Dutch Ministry of Agriculture, Nature and Food Quality that aims to develop alternatives to animal use. We are grateful to Olivier Burry for preparing the script and his advice on the nearest neighbor analysis. 